\title{
Identification of Pentatricopeptide Repeat Proteins in the Model Organism Dictyostelium discoideum
}

\author{
Sam Manna, Jessica Brewster, and Christian Barth \\ Department of Microbiology, Thomas Cherry Building, La Trobe University, Kingsbury Drive, Bundoora, VIC 3086, Australia \\ Correspondence should be addressed to Christian Barth; c.barth@latrobe.edu.au
}

Received 18 April 2013; Accepted 11 July 2013

Academic Editor: Brian Wigdahl

Copyright (c) 2013 Sam Manna et al. This is an open access article distributed under the Creative Commons Attribution License, which permits unrestricted use, distribution, and reproduction in any medium, provided the original work is properly cited.

Pentatricopeptide repeat (PPR) proteins are RNA binding proteins with functions in organelle RNA metabolism. They are found in all eukaryotes but have been most extensively studied in plants. We report on the identification of 12 PPR-encoding genes in the genome of the protist Dictyostelium discoideum, with potential homologs in other members of the same lineage and some predicted novel functions for the encoded gene products in protists. For one of the gene products, we show that it localizes to the mitochondria, and we also demonstrate that antisense inhibition of its expression leads to slower growth, a phenotype associated with mitochondrial dysfunction.

\section{Introduction}

Mitochondria contain their own genome and, as is the case for any other genome, must maintain tight control over the expression of their encoded gene products. Mitochondrial genes typically encode either components of the respiratory chain for ATP synthesis or the mitochondrial translation machinery. Regulating the expression of such genes is therefore essential for normal cell function, as aberrations in the regulation of mitochondrial gene expression can result in disease $[1,2]$. Similarly to nuclear and bacterial gene expression, post-transcriptional regulation is one of the most important stages of mitochondrial gene expression. This can include processing of polycistronic transcripts and liberation of structural RNAs, excision of introns, RNA editing, and stability modifications such as polyadenylation [2].

Given that these post-transcriptional processes are highly diverse, one would expect such functions to be catalysed by many different proteins. Indeed, each post-transcriptional event often involves several proteins, amongst which a large family of helical repeat proteins have been found to play important roles in organelle gene expression. These rather complex proteins are known as pentatricopeptide repeat (PPR) proteins and were originally identified during the sequencing of the genome of the model plant Arabidopsis thaliana [3]. The PPR family is now known as one of the largest protein families to exist in angiosperms with over 450 PPR-encoding genes identified in A. thaliana [4].

PPR proteins are characterised by a 35 amino acid motif, often repeated in tandem a variable number of times [3, 5]. Each PPR motif consists of two antiparallel $\alpha$-helices, which interact with each other $[3,5]$. The series of $\alpha$-helices form a superhelix containing a groove, which can bind its RNA ligand in a sequence-specific manner [5-7]. Most PPR proteins function as molecular adaptors in the recruitment of catalytic enzymes or effector proteins to target transcripts $[5,7]$. Two classes of PPR proteins exist. The $\mathrm{P}$ class is characterised by the canonical 35 amino acid motif and typically lacks additional domains [5]. The second class, the PLS class, consists of slightly longer and shorter PPR motifs, as well as C-terminal domains such as the $\mathrm{E}, \mathrm{E}+$, and DYW domains, which often have prominent roles in RNA editing [5]. Indeed, the presence of PLS class PPR proteins, originally believed to be exclusive to plants, correlates strongly with the occurrence of organelle RNA editing, while these proteins are typically absent in organisms where organelle RNA editing does not occur $[8,9]$. Although not as prevalent as in plants, PPR proteins are found in all eukaryotes, where they have specific roles in post-transcriptional regulation of organelle gene expression. Such functions include processing, splicing, RNA editing, stabilisation, polyadenylation, and translational 
activation [5, 7]. Although several of these functions are regulated by PPR proteins in plants, the most common function for plant PPR proteins seems to be in RNA editing, a process which is rather common in plant organelles $[5,10]$. In humans, only seven PPR proteins have been identified. They have been shown to regulate the mitochondrial transcriptome not via RNA editing, but rather through transcription and transcript processing, RNA stability, polyadenylation, and translation [11-15].

While the knowledge of PPR protein structure and function in non-plant organisms is expanding exponentially, little is known about the significance of these proteins in the mitochondria of protozoa. In the protists, PPR proteins have been studied mainly in trypanosomatids, where more than 30 PPR genes have been identified, a uniquely high number for a non-plant organism [16-19]. Most of these PPR proteins play roles in either the stabilisation or polyadenylation of kinetoplast transcripts, and they often lack additional Cterminal domains [16-19]. While studies into the heterolobosean protist Naegleria gruberi have also identified an unexpectedly high number of PPR-encoding genes, in contrast to trypanosomes a large subset of the gene products belongs to the DYW subclass of the PLS group and has thus been implicated in RNA editing [20, 21]. Despite the identification of PPR genes in N. gruberi, none of their gene products have been functionally characterised, and therefore the question remains whether transcript stabilisation and editing are the main functions of PPR proteins in protists.

Dictyostelium discoideum is a cellular slime mould belonging to the Amoebozoa and is a widely accepted and wellestablished model for studying mitochondrial genetics and disease $[22,23]$. Transcription of the mitochondrial genome in $D$. discoideum has been studied in detail, and some of the core components mediating the transcription process have been identified. In $D$. discoideum mitochondria, transcription is initiated at a single site and the transcriptome is subjected to several post-transcriptional modifications including processing and intron splicing, as well as a single nucleotide RNA editing event that occurs in the transcript of the mitochondrial rns gene [24-28]. However, very little is known about the proteins that regulate these post-transcriptional events, and the existence and potential role of PPR proteins in mitochondrial RNA metabolism have not been investigated in this organism. Here, we describe the identification of genes of the PPR protein family in $D$. discoideum. We found 12 potential PPR proteins encoded in the $D$. discoideum genome, and some of these proteins show significantly different features compared to other known PPR proteins. One of the D. discoideum proteins has been characterised in detail, confirming its mitochondrial localisation. We also demonstrate that antisense inhibition of its expression leads to growth defects, a phenotype associated with mitochondrial dysfunction. While the phenotypic changes resulting from antisense inhibition of gene expression of one of these PPR proteins confirm the importance of these proteins in mitochondrial function, their specific role in post-transcriptional regulation of the $D$. discoideum mitochondrial transcriptome still remains to be determined.

\section{Materials and Methods}

2.1. Strains and Culture Conditions. D. discoideum strain AX2 and all transformants were grown to a density of $2-5 \times 10^{6}$ cells/mL in HL-5 medium at $21^{\circ} \mathrm{C}[29,30]$. For non-axenic culture, AX2 and all derivatives were grown on SM plates with Klebsiella aerogenes lawns [31] unless otherwise stated.

2.2. Transformation of D. discoideum with Vector DNA. The calcium phosphate precipitation method was used to transform $D$. discoideum with vector DNA as described previously [32] using $20 \mu \mathrm{g}$ of vector DNA. Transformants were isolated on Micrococcus luteus lawns on SM plates supplemented with $20 \mu \mathrm{g} / \mathrm{mL}$ G-418. [33].

2.3. Fluorescence Microscopy. To determine the subcellular localisation of $\mathrm{PtcB}, \mathrm{D}$. discoideum transformants expressing a PtcB:GFP fusion protein were analysed via fluorescence microscopy as described previously [34, 35]. Aliquots of the axenically grown transformant culture $(\sim 3 \mathrm{~mL})$ were transferred into a 6-well plate (BD Biosciences) containing coverslips, and the cells were allowed to settle. The medium was removed and the mitochondria were stained with $100 \mathrm{nM}$ MitoTracker (Life Technologies) in Lo-Flo HL-5 medium for 1 hour. Unbound MitoTracker was removed by washing the cells four times with Lo-Flo HL-5 and twice with phosphate buffer. The cells were subsequently fixed by placing the coverslips for 15 minutes upside down onto a $1 \%$ agarose gel in phosphate buffer containing 3.7\% paraformaldehyde, after which the cells were washed four times with phosphate buffered saline (PBS). Coverslips were rinsed with Milli-Q $\mathrm{sdH}_{2} \mathrm{O}$ and mounted for microscopy with $90 \%$ glycerol in PBS.

2.4. Analysis of Growth Rates on Bacterial Lawns. Growth of $D$. discoideum cells was analysed by measuring plaque expansion rates on bacterial lawns as described previously [36]. Briefly, D. discoideum cells of interest were collected from the leading edge of a previously grown plaque on $K$. aerogenes lawns. The cells were then used to inoculate normal agar plates with pregrown Escherichia coli B2 lawns. The diameter of the plaques was measured every 8 or 16 hours for 7 days to calculate the mean plaque expansion rate ( $\mathrm{mm}$ /hour) as an estimate of growth.

2.5. Quantitative PCR. The number of vector copies of the $p t c B$ antisense construct in each transformant was determined using qPCR. The qPCR reactions were performed using SsoAdvanced SYBR Green Supermix (Bio-Rad). Total gDNA extracted from each antisense transformant and from wild type cells was used as template along with primers specific to the cloned portion of the $p t c B$ gene. Cycling conditions were as follows: initial denaturation at $95^{\circ} \mathrm{C}$ for 10 minutes and then 40 cycles of denaturation at $95^{\circ} \mathrm{C}$ for 15 seconds, followed by annealing and primer extension at $60^{\circ} \mathrm{C}$ for 1 minute. All transcript levels were normalised to the single copy number $\beta$-tubulin $(t u b B)$ gene. 
TABLE 1: Bioinformatic analysis of putative $D$. discoideum pentatricopeptide repeat candidates. The probability of the helical repeats being pentatricopeptide repeats and the number of motifs were predicted using TPRpred, and the probability of mitochondrial targeting was predicted using Mitoprot.

\begin{tabular}{|c|c|c|c|c|c|c|}
\hline \multicolumn{3}{|c|}{ Gene information } & \multicolumn{4}{|c|}{ Protein information } \\
\hline Gene & $\begin{array}{l}\text { Chromosome } \\
\text { location }\end{array}$ & Gene size (bp) & $\begin{array}{c}\text { Length } \\
\text { (amino acids) }\end{array}$ & $\begin{array}{l}\text { Probability } \\
\text { of PPR (\%) }\end{array}$ & $\begin{array}{l}\text { Number of } \\
\text { PPR motifs }\end{array}$ & $\begin{array}{c}\text { Probability of mitochondrial } \\
\text { targeting (\%) }\end{array}$ \\
\hline$p t c A$ & 6 & 1,518 & 505 & 99.99 & 4 & 98 \\
\hline$p t c B$ & 5 & 1,783 & 528 & 100 & 9 & 91 \\
\hline$p t c C$ & 5 & 2,076 & 611 & 100 & 11 & 80 \\
\hline$p t c D$ & 5 & 4,321 & 1,405 & 97.18 & 7 & 23 \\
\hline$p t c E$ & 1 & 2,334 & 746 & 100 & 5 & 53 \\
\hline$p t c F$ & 3 & 1,596 & 531 & 100 & 6 & 93 \\
\hline$p t c G$ & 2 & 1,371 & 423 & 100 & 5 & 92 \\
\hline$p t c H$ & 3 & 3,247 & 1,057 & 96.37 & 4 & 88 \\
\hline$p t c I$ & 2 & 3,737 & 1,163 & 96.62 & 10 & 89 \\
\hline$p t c J$ & 2 & 3,868 & 1,258 & 53.44 & 5 & 95 \\
\hline$p t c K$ & 2 & 3,623 & 1,148 & 100 & 11 & 54 \\
\hline$p t c L$ & 6 & 3,351 & 1,116 & 8.28 & 6 & 66 \\
\hline
\end{tabular}

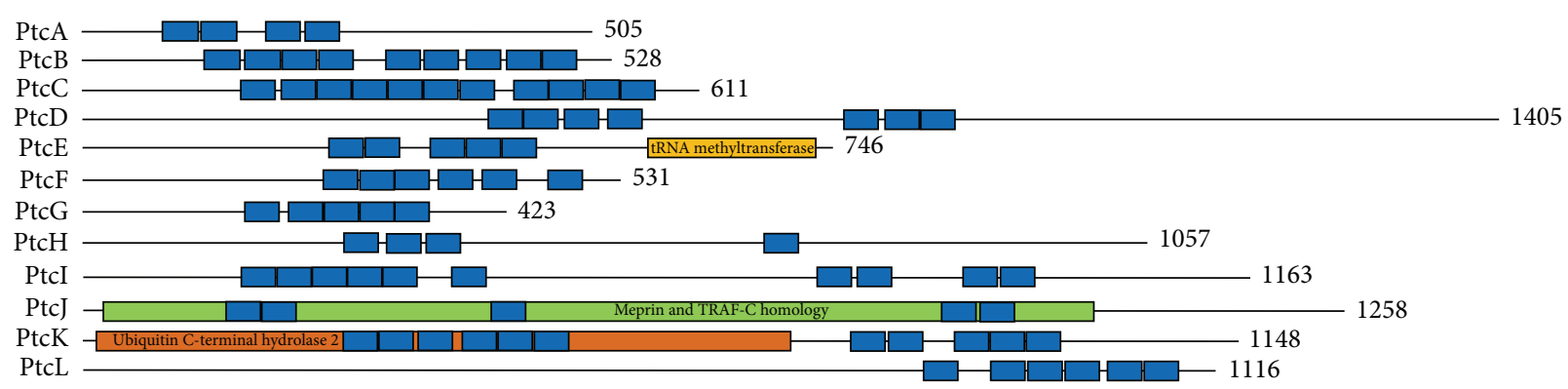

FIgURE 1: Predicted domain architecture of $D$. discoideum PPR proteins, PtcA-L. Blue boxes represent PPR motifs. The amino acid length of each protein is indicated at the C-terminus of each protein. Also displayed are the putative tRNA methyltransferase (yellow), MATH-like (green), and ubiquitin hydrolase-like (orange) domains of PtcE, PtcJ, and PtcK, respectively.

\section{Results and Discussion}

3.1. Identification of PPR Proteins in D. discoideum. We analysed the D. discoideum genome for any PPR-encoding genes and identified 12 gene sequences coding for putative helical repeat containing proteins. Analysis of the protein sequences using the bioinformatics tool TPRpred [37] confirmed that all candidates contained putative PPR motifs (Table 1). The candidates were named pentatricopeptide repeat containing proteins A-L (PtcA-L). They range in size from 423 to 1405 amino acids, and based on the TPRpred analysis, each contains anywhere from 4 to 11 canonical P class PPR motifs, a typical range for a non-plant PPR protein. The number of PPR proteins identified in $D$. discoideum was also consistent with that observed in other non-plant eukaryotes but was significantly less than the number of PPR proteins observed in other protists such as trypanosomatids and heterolobosea. We did not identify any PLS class-specific features in the PPR protein candidates (Figure 1). The lack of PLS class PPR proteins in D. discoideum suggests that PPR proteins are not involved in RNA editing, which correlates well with the rather infrequent occurrence of editing in $D$. discoideum mitochondrial transcripts. This is in contrast to plants and $N$. gruberi, which contain PLS class PPR proteins known to be involved in RNA editing [5, 20, 21].

Although most of the identified PPR proteins appear to lack any additional C-terminal domains, one candidate, PtcE, contains a putative C-terminal tRNA $\mathrm{m}^{7} \mathrm{G} 46$ methyltransferase domain. PtcE is, therefore, predicted to catalyse the methylation of mitochondrial tRNA species which contain a guanosine residue at position 46 , a role that has not previously been reported for any other PPR protein.

PtcK has a putative ubiquitin carboxyl-terminal hydrolase 2 domain. However, it is noteworthy that PtcK only displays weak similarity to ubiquitin hydrolases and thus may contain a non-functional domain or a similar sequence by chance. Although not homologous, PtcK exhibits similarity to several members of a PPR-like family of plant organelle RNA binding proteins (Figure 2), which contain a plant organelle RNA recognition (PORR) domain (formerly known as domain of unknown function 860 or DUF860). These RNA binding domains are thought to be exclusive to 


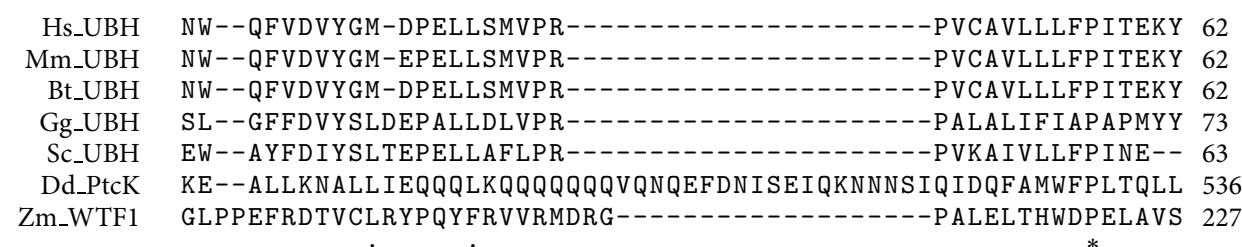

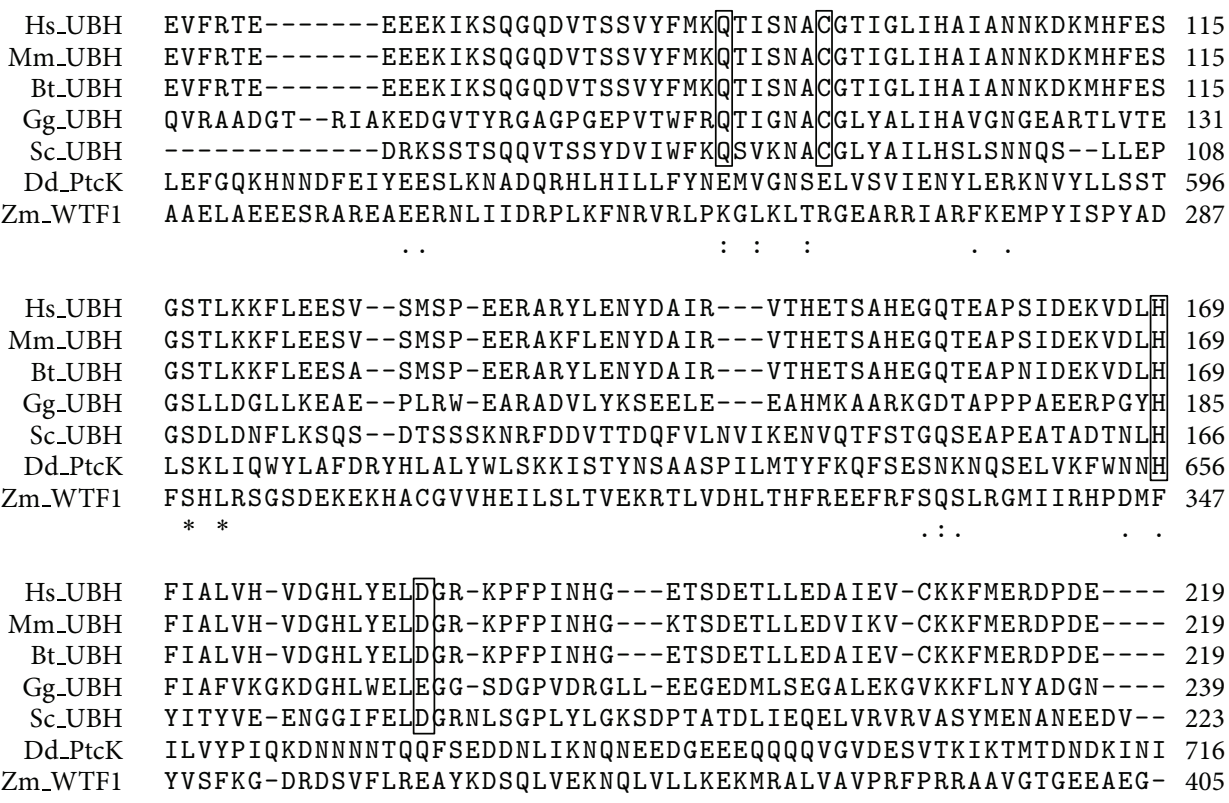

FIgURe 2: Amino acid sequence alignment of PtcK from $D$. discoideum (Dd) with ubiquitin hydrolases (UBHs) from other organisms. Sequences used in the alignment include UBHs from Homo sapiens (Hs, accession number NP_005993), Mus musculus (Mm, accession number AAF64193), Bos Taurus (Bt, accession number NP_001035631), Glomerella graminicola (Gg, accession number EFQ25707), Saccharomyces cerevisiae (Sc, accession number EDN63415), and WTF1, a PORR-containing protein from Zea mays (Zm, accession number ACI96105). Only the relevant portion of the alignment is shown. Boxed residues indicate conserved amino acids required for ubiquitin hydrolase activity while identical (*), conserved (:), and semiconserved (.) amino acids are also denoted.

plants, and like the domain in PtcK they not only display weak similarity to ubiquitin hydrolases, but also lack most of the catalytic residues (Figure 2 ) required for such activity $[38,39]$. Additionally, the RNA binding surface of PORR proteins is similar to that of repeated helical motifs, such as PPR motifs, and they have been shown to mediate several aspects of organelle gene expression at the RNA level [38, 39]. Only two members of this family have been characterised and both mediate splicing of introns in organelle transcripts $[38,40]$. Although PtcK may not be a member of this family, the features it has in common with the PORR family in addition to the presence of PPR motifs not only imply a similar function for PtcK in mitochondrial gene expression, but also demonstrate a potential evolutionary link between PPR proteins and the PORR family. In fact, the latter may not be restricted to plants as originally postulated, as PtcK clearly demonstrates that proteins similar to the PORR family exist outside of the plant lineage.

Another PPR protein candidate, PtcJ is predicted to contain a meprin and TRAF-C homology (MATH) domain, a domain involved in peptide cleavage and processing, signal transduction, and ubiquitination [41]. However, given that these are unlikely functions for a PPR protein and that the similarity of PtcJ to the MATH domain is weak, PtcJ may exhibit a scenario similar to PtcK in that the MATH domain is not catalytic, but rather may be an RNA binding domain.

Lastly, TPRpred analysis of PtcL provided a low probability of the candidate being a PPR protein (Table 1), despite the fact that there were at least six PPR motifs, and no other features were detected. It is, therefore, important to note that in previous work in Trypanosoma brucei, a PPR candidate (TbPPR9) had been identified with a TPRpred score even lower than that obtained for PtcL, but the T. brucei protein was later shown to be a bona fide PPR protein [19]. Considering this, and taking into account the degenerate nature of PPR motifs, it is not unreasonable to postulate that PtcL, despite its low probability score, may also be a bona fide PPR protein.

3.2. A D. discoideum PPR Candidate Localizes to Mitochondria. Additional in silico analysis of the protein sequences indicated that most of these candidates are predicted to contain N-terminal mitochondrial targeting signals (Table 1) as inferred by the software program Mitoprot [42]. Following their initial identification, one PPR candidate, PtcB, was selected for further analysis. To confirm its mitochondrial 


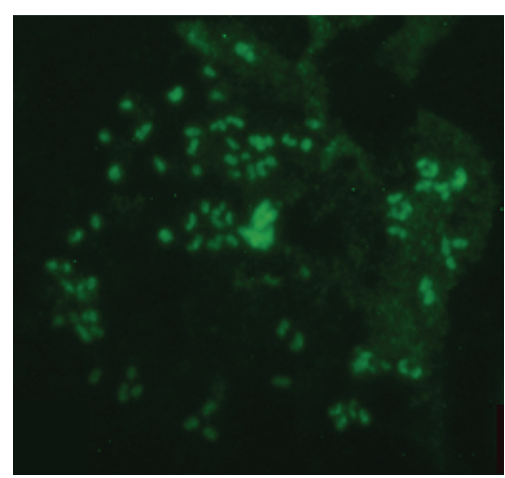

(a)

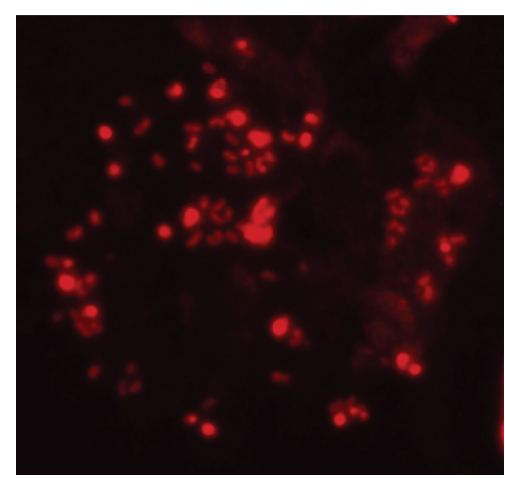

(b)

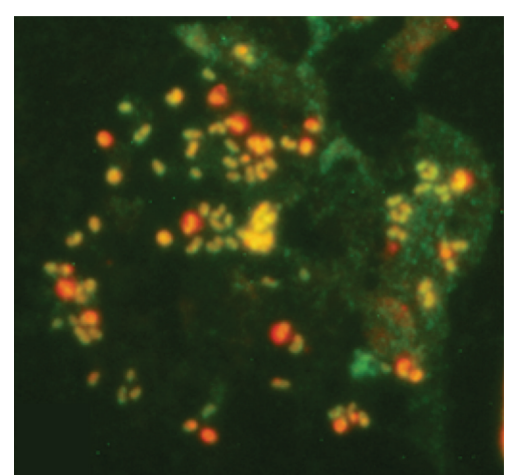

(c)

FIgURE 3: Subcellular localisation of PtcB. Fluorescence microscopy of D. discoideum cells (a) expressing a PtcB:GFP fusion protein,(b) stained with Mitotracker, (c) indicating that the fusion protein and the mitochondria colocalise.

localisation, a fusion gene was created containing the $5^{\prime}$ end of the $p t c B$ gene ( $414 \mathrm{bp}$ ), encoding the putative mitochondrial targeting signal, fused to the gene encoding the green fluorescent protein (GFP). When this construct was transformed and expressed in $D$. discoideum cells, the PtcB:GFP fusion protein colocalised with the mitochondria (Figure 3), confirming that PtcB is indeed a mitochondrial protein and suggesting a physiological role for the protein within this organelle.

\subsection{Antisense Inhibition of D. discoideum PPR Expression} Results in Slower Growth, a Phenotype Associated with Mitochondrial Dysfunction. To confirm a functional role of the D. discoideum PPR protein PtcB in the mitochondria, the expression of $p t c B$ was knocked down via antisense inhibition. This involved cloning a portion of the $p t c B$ gene (414 bp) into the $D$. discoideum expression vector $\mathrm{pDNeo} 2$ [43] in the antisense orientation relative to the actin 6 promoter. Expression of the $p t c B$ gene fragment from this promoter will synthesise an antisense RNA transcript complementary to the endogenous $p t c B$ mRNA sequence. Upon transformation of $D$. discoideum with vector DNA, the expression vector randomly integrates into the genome, whereby a single founding vector molecule will replicate at the integration site creating a random number of multimers [44]. As a result of this unique co-insertional replication mechanism, each D. discoideum transformant contains a different number of copies of the antisense construct and consequently, each transformant exhibits a different level of antisense inhibition [27]. This feature allows the antisense inhibition of a gene in a dosage-dependent manner. Following transformation of $D$. discoideum cells with the $p t c B$ antisense construct, 13 antisense transformants were isolated. To establish whether PtcB has an essential role in mitochondrial function, the growth rates for these transformants were determined by growing the transformants on bacterial lawns. In $D$. discoideum, growth has been demonstrated to be one of the first phenotypes affected by non-functioning mitochondria, and thus slower growth serves as an indicator of mitochondrial dysfunction $[27,36]$. This is because mitochondrial dysfunction triggers a cascade of pathways in $D$. discoideum that favour the

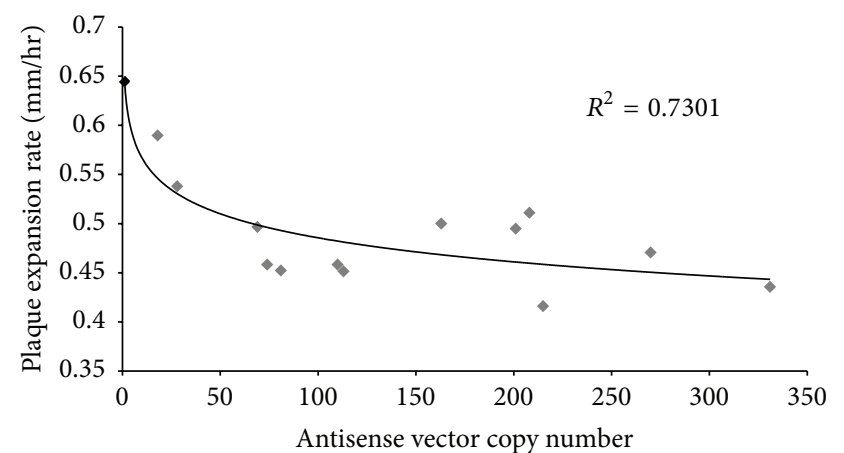

FIGURE 4: Plaque expansion rates of $p t c B$ antisense transformants on Escherichia coli B2 lawns. Plaque expansion rates for $p t c B$ antisense transformants are plotted against the copy number of the antisense construct present in each transformant, a reflection of the level of antisense inhibition. The number of copies of the antisense construct in each transformant was determined using qPCR. All transformants are shaded in grey while the wild type parental strain is in black.

repression of ATP consuming processes such as growth [27, 36]. Antisense inhibition of $p t c B$ resulted in slower plaque expansion rates on bacterial lawns, and the severity of this phenotype correlated with the level of antisense inhibition of $p t c B$ as indicated by the number of antisense constructs present in each of the transformants (Figure 4). The slower growth of $D$. discoideum antisense transformants confirms the important role PPR proteins play in D. discoideum mitochondrial function. Delayed growth upon knockdown of PPR-encoding genes has also been observed in trypanosomes $[18,19]$, and in plants, PPR mutants are known to display phenotypes associated with chloroplast or mitochondrial dysfunction, including cytoplasmic male sterility, negative effects on embryonic development, and defective photosynthetic ability $[5,45,46]$.

3.4. D. discoideum PPR Proteins Possess Homologs in the Cellular Slime Mould Lineage. To gain further insight into the evolution of PPR proteins in the cellular slime mould 
TABLE 2: Putative homologs of $D$. discoideum PPR proteins in other cellular slime moulds. The presence of a homolog is noted by the NCBI protein accession number while the absence of a clear homolog is denoted by "-.". Also, indicated in the parentheses are the levels of amino acid identity/similarity (\%), respectively, for each protein compared to the $D$. discoideum homolog as determined by end to end pairwise alignments.

\begin{tabular}{|c|c|c|c|}
\hline $\begin{array}{l}\text { D. discoideum } \\
\text { protein }\end{array}$ & $\begin{array}{l}\text { D. purpureum } \\
\text { homolog }\end{array}$ & $\begin{array}{l}\text { P. pallidum } \\
\text { homolog }\end{array}$ & $\begin{array}{l}\text { D. fasciculatum } \\
\text { homolog }\end{array}$ \\
\hline PtcA & $\begin{array}{c}\text { XP_003289503 } \\
(26.5 / 43.6)\end{array}$ & - & - \\
\hline PtcB & $\begin{array}{c}\text { XP_003288427 } \\
(52.9 / 71.2)\end{array}$ & $\begin{array}{c}\text { EFA79424 } \\
(16 / 22.7)\end{array}$ & $\begin{array}{l}\text { EGG14329 } \\
(32.1 / 49.4)\end{array}$ \\
\hline PtcC & $\begin{array}{c}\text { XP_003290170 } \\
(48.1 / 66.6)\end{array}$ & $\begin{array}{l}\text { EFA76720 } \\
(37.4 / 55.5)\end{array}$ & $\begin{array}{c}\text { EGG22645 } \\
(39.3 / 62.1)\end{array}$ \\
\hline PtcD & $\begin{array}{c}\text { XP_003284803 } \\
(21.7 / 33.1)\end{array}$ & - & - \\
\hline PtcE & $\begin{array}{c}\text { XP_003288663 } \\
(67.5 / 78.5)\end{array}$ & $\begin{array}{c}\text { EFA82229 } \\
(46.3 / 60.9)\end{array}$ & $\begin{array}{c}\text { EGG13534 } \\
(17.5 / 24.6)\end{array}$ \\
\hline PtcF & $\begin{array}{c}\text { XP_003294037 } \\
(49.9 / 64.8)\end{array}$ & $\begin{array}{l}\text { EFA79525 } \\
(27.5 / 50.3)\end{array}$ & $\begin{array}{c}\text { EGG15096 } \\
(29 / 49)\end{array}$ \\
\hline PtcG & $\begin{array}{c}\text { XP_003284179 } \\
(61.9 / 75.1)\end{array}$ & $\begin{array}{c}\text { EFA75260 } \\
(28.2 / 37)\end{array}$ & $\begin{array}{l}\text { EGG14213 } \\
(49.5 / 64.8)\end{array}$ \\
\hline $\mathrm{PtcH}$ & $\begin{array}{c}\text { XP_003286839 } \\
(24.6 / 41.2)\end{array}$ & - & - \\
\hline PtcI & $\begin{array}{c}\text { XP_003285976 } \\
(24.4 / 41.5)\end{array}$ & - & - \\
\hline PtcJ & $\begin{array}{c}\text { XP_003291714 } \\
(25.6 / 44.5)\end{array}$ & - & - \\
\hline PtcK & $\begin{array}{c}\text { XP_003293255 } \\
(27.2 / 44.3)\end{array}$ & - & - \\
\hline PtcL & $\begin{array}{c}\text { XP_003286762 } \\
(25.8 / 42.2)\end{array}$ & - & - \\
\hline
\end{tabular}

lineage, we searched for PPR protein-encoding genes in the genomes of three other cellular slime moulds, Dictyostelium purpureum, Dictyostelium fasciculatum, and Polysphondylium pallidum. Interestingly, the search led to the identification of what seemed to be homologs of most of the PPR proteins previously identified in $D$. discoideum (Table 2). For most of these homologs it was confirmed by TPRpred analysis that they contain PPR motifs (Table 3). In two of the proteins, however, PPR motifs could not be detected (protein accession numbers XP_003284803 and XP_003286762), despite the fact that each of the candidates displayed a high level of homology to a specific $D$. discoideum PPR protein (Table 2). The failure to identify any PPR motifs within these proteins may be a result of weak conservation of their PPR motifs.

None of the identified PPR proteins seem to have homologs in organisms outside of the cellular slime mould lineage (data not shown). A similar pattern of high conservation of PPR homologs has also been observed previously for nonplant PPR proteins in closely related species [17, 19]. The high level of conservation not only demonstrates the importance of PPR proteins in mitochondrial function, but also suggests a specific role for each of these homologs. It is therefore likely that these proteins fulfil more similar functions required by
TABLE 3: Bioinformatic analysis of $D$. discoideum PPR protein homologs in other cellular slime moulds. The probability of helical repeats being PPR and the predicted number of motifs were determined using TPRpred.

\begin{tabular}{lccc}
\hline Organism & $\begin{array}{c}\text { NCBI protein } \\
\text { accession number }\end{array}$ & $\begin{array}{c}\text { PPR } \\
\text { probability (\%) }\end{array}$ & $\begin{array}{c}\text { Number of } \\
\text { PPR motifs }\end{array}$ \\
\hline D. purpureum & XP_003289503 & 100 & 9 \\
D. purpureum & XP_003288427 & 100 & 9 \\
D. purpureum & XP_003290170 & 100 & 12 \\
D. purpureum & XP_003284803 & 0 & 0 \\
D. purpureum & XP_003288663 & 100 & 5 \\
D. purpureum & XP_003294037 & 100 & 6 \\
D. purpureum & XP_003284179 & 100 & 5 \\
D. purpureum & XP_003286839 & 100 & 9 \\
D. purpureum & XP_003285976 & 100 & 15 \\
D. purpureum & XP_003291714 & 0.89 & 3 \\
D. purpureum & XP_003293255 & 99.96 & 9 \\
D. purpureum & XP_003286762 & 0 & 0 \\
P. pallidum & EFA79424 & 100 & 9 \\
P. pallidum & EFA76720 & 100 & 13 \\
P. pallidum & EFA82229 & 100 & 3 \\
P. pallidum & EFA79525 & 100 & 8 \\
P. pallidum & EFA75260 & 100 & 5 \\
D. fasciculatum & EGG14329 & 100 & 9 \\
D. fasciculatum & EGG22645 & 100 & 12 \\
D. fasciculatum & EGG13534 & 97.20 & 3 \\
D. fasciculatum & EGG15096 & 100 & 8 \\
D. fasciculatum & EGG14213 & 100 & 6 \\
\hline
\end{tabular}

TABLE 4: Bioinformatic analysis of unique PPR proteins found in one, but not in other cellular slime moulds. The probability of helical repeats being PPR and the predicted number of motifs were determined using TPRpred.

\begin{tabular}{lccc}
\hline Organism & $\begin{array}{c}\text { NCBI protein } \\
\text { accession number }\end{array}$ & $\begin{array}{c}\text { PPR } \\
\text { probability (\%) }\end{array}$ & $\begin{array}{c}\text { Number of } \\
\text { PPR motifs }\end{array}$ \\
\hline D. purpureum & XP_003291713 & 99.55 & 8 \\
P. pallidum & EFA82227 & 64.34 & 3 \\
P. pallidum & EFA76758 & 51.88 & 6 \\
P. pallidum & EFA80531 & 100 & 15 \\
D. fasciculatum & EGG19875 & 99.98 & 8 \\
D. fasciculatum & EGG23890 & 100 & 12 \\
\hline
\end{tabular}

all four cellular slime mould species. However, some PPR homologs could only be found in $D$. discoideum and $D$. purpureum, indicating a potential conserved function of the proteins in these organisms, which is either not required or is performed by a different protein in P. pallidum and D. fasciculatum mitochondria. In addition, our sequence analysis also revealed that some of the cellular slime moulds possess PPR proteins which are not found in any of the others (Table 4). These candidates may represent unique PPR proteins that perform functions only required in these 
cellular slime moulds. However, it is noteworthy to mention that one of these proteins, XP_003291713 from D. purpureum, may have a putative homolog in $D$. discoideum (protein accession number XP_644522), but no PPR motifs were detected in the D. discoideum protein by TPRpred (data not shown).

\section{Conclusions}

The presence of PPR proteins in the model eukaryote $D$. discoideum suggests an important role for these proteins in the regulation of the mitochondrial transcriptome. This is supported by the antisense inhibition of one of the PPRencoding genes, $p t c B$, yielding phenotypes characteristic of mitochondrial dysfunction in the protist. While the precise function of PPR proteins remains to be elucidated, it is clear that the function of most of these proteins is conserved supported by the presence of homologs in other cellular slime moulds. The potential functions of these proteins seem to differ from the function of RNA editing type PPR proteins in $N$. gruberi but may be analogous to the function of trypanosomal PPR proteins in modifying the stability of mitochondrial transcripts. One of the PPR candidates identified, PtcE, also contains a C-terminal methyltransferase domain, which has not been identified in any PPR protein to date, further attesting to the significance of studying PPR proteins in the $D$. discoideum model. The potential methyltransferase activity and the presence of other domains in some of the PPR proteins, therefore, suggest some unique functions for PPR proteins in $D$. discoideum mitochondria which have not been observed for PPR proteins of other organisms before. Thus, the functional study of PPR proteins in $D$. discoideum will provide an elegant system for investigating the important role PPR proteins played not only in protozoan mitochondrial gene expression but also more generally in non-plant organisms.

\section{Abbreviations}

PPR: Pentatricopeptide repeat

PtcA-L: Pentatricopeptide repeat-containing protein A-L

PORR: Plant organelle RNA recognition.

\section{Conflict of Interests}

All authors declare that they do not have any conflict of interests with any trademark or software mentioned in this paper.

\section{Acknowledgment}

Sam Manna was the recipient of an Australian Postgraduate Award.

\section{References}

[1] M. W. Gray, B. F. Lang, and G. Burger, "Mitochondria of protists," Annual Review of Genetics, vol. 38, pp. 477-524, 2004.

[2] T. E. Shutt and G. S. Shadel, "A compendium of human mitochondrial gene expression machinery with links to disease,"
Environmental and Molecular Mutagenesis, vol. 51, no. 5, pp. 360-379, 2010.

[3] I. D. Small and N. Peeters, "The PPR motif-a TPR-related motif prevalent in plant organellar proteins," Trends in Biochemical Sciences, vol. 25, no. 2, pp. 46-47, 2000.

[4] C. Lurin, C. Andrés, S. Aubourg et al., "Genome-wide analysis of Arabidopsis pentatricopeptide repeat proteins reveals their essential role in organelle biogenesis," Plant Cell, vol. 16, no. 8, pp. 2089-2103, 2004.

[5] C. Schmitz-Linneweber and I. Small, "Pentatricopeptide repeat proteins: a socket set for organelle gene expression," Trends in Plant Science, vol. 13, no. 12, pp. 663-670, 2008.

[6] J. Pfalz, O. A. Bayraktar, J. Prikryl, and A. Barkan, "Site-specific binding of a PPR protein defines and stabilizes $5^{\prime}$ and $3^{\prime}$ mRNA termini in chloroplasts," The EMBO Journal, vol. 28, no. 14, pp. 2042-2052, 2009.

[7] E. Delannoy, W. A. Stanley, C. S. Bond, and I. D. Small, "Pentatricopeptide repeat (PPR) proteins as sequence-specificity factors in post-transcriptional processes in organelles," Biochemical Society Transactions, vol. 35, no. 6, pp. 1643-1647, 2007.

[8] M. Rüdinger, M. Polsakiewicz, and V. Knoop, "Organellar RNA editing and plant-specific extensions of pentatricopeptide repeat proteins in jungermanniid but not in marchantiid liverworts," Molecular Biology and Evolution, vol. 25, no. 7, pp. 14051414, 2008.

[9] V. Salone, M. Rüdinger, M. Polsakiewicz et al., "A hypothesis on the identification of the editing enzyme in plant organelles," FEBS Letters, vol. 581, no. 22, pp. 4132-4138, 2007.

[10] S. Fujii and I. Small, "The evolution of RNA editing and pentatricopeptide repeat genes," New Phytologist, vol. 191, no. 1, pp. 37-47, 2011.

[11] O. Rackham and A. Filipovska, "The role of mammalian PPR domain proteins in the regulation of mitochondrial gene expression," Biochimica et Biophysica Acta, vol. 1819, no. 9-10, pp. 1008-1016, 2011.

[12] O. Rackham, T. R. Mercer, and A. Filipovska, "The human mitochondrial transcriptome and the RNA-binding proteins that regulate its expression," Wiley Interdisciplinary Reviews RNA, vol. 3, no. 5, pp. 675-695, 2012.

[13] M. I. G. L. Sanchez, T. R. Mercer, S. M. K. Davies et al., "RNA processing in human mitochondria," Cell Cycle, vol. 10, no. 17, pp. 2904-2916, 2011.

[14] S. M. K. Davies, O. Rackham, A.-M. J. Shearwood et al., "Pentatricopeptide repeat domain protein 3 associates with the mitochondrial small ribosomal subunit and regulates translation," FEBS Letters, vol. 583, no. 12, pp. 1853-1858, 2009.

[15] S. M. Davies, M. I. L. Sanchez, R. Narsai et al., "MRPS27 is a pentatricopeptide repeat domain protein required for the translation of mitochondrially encoded proteins," FEBS Letters, vol. 586, no. 20, pp. 3555-3561, 2012.

[16] I. Aphasizheva, D. Maslov, X. Wang, L. Huang, and R. Aphasizhev, "Pentatricopeptide repeat proteins stimulate mrna adenylation/uridylation to activate mitochondrial translation in trypanosomes," Molecular Cell, vol. 42, no. 1, pp. 106-117, 2011.

[17] M. K. Mingler, A. M. Hingst, S. L. Clement, L. E. Yu, L. Reifur, and D. J. Koslowsky, "Identification of pentatricopeptide repeat proteins in Trypanosoma brucei," Molecular and Biochemical Parasitology, vol. 150, no. 1, pp. 37-45, 2006.

[18] M. Pusnik, I. Small, L. K. Read, T. Fabbro, and A. Schneider, "Pentatricopeptide repeat proteins in Trypanosoma brucei function in mitochondrial ribosomes," Molecular and Cellular Biology, vol. 27, no. 19, pp. 6876-6888, 2007. 
[19] M. Pusnik and A. Schneider, "A trypanosomal pentatricopeptide repeat protein stabilizes the mitochondrial mRNAs of cytochrome oxidase subunits 1 and 2," Eukaryotic Cell, vol. 11, no. 1, pp. 79-87, 2012.

[20] V. Knoop and M. Rüdinger, "DYW-type PPR proteins in a heterolobosean protist: plant RNA editing factors involved in an ancient horizontal gene transfer?" FEBS Letters, vol. 584, no. 20, pp. 4287-4291, 2010.

[21] M. Rüdinger, L. Fritz-Laylin, M. Polsakiewicz, and V. Knoop, "Plant-type mitochondrial RNA editing in the protist Naegleria gruberi," RNA, vol. 17, no. 12, pp. 2058-2062, 2011.

[22] S. J. Annesley and P. R. Fisher, "Dictyostelium discoideum-a model for many reasons," Molecular and Cellular Biochemistry, vol. 329, no. 1-2, pp. 73-91, 2009.

[23] L. M. Francione, S. J. Annesley, S. Carilla-Latorre, R. Escalante, and P. R. Fisher, "The Dictyostelium model for mitochondrial disease," Seminars in Cell and Developmental Biology, vol. 22, no. 1, pp. 120-130, 2011.

[24] K. Angata, S. Ogawa, K. Yanagisawa, and Y. Tanaka, "A groupI intron in the mitochondrial large-subunit ribosomal RNAencoding gene of Dictyostelium discoideum: same site localization in alga and in vitro self-splicing," Gene, vol. 153, no. 1, pp. 49-55, 1995.

[25] C. Barth, U. Greferath, M. Kotsifas, and P. R. Fisher, "Polycistronic transcription and editing of the mitochondrial small subunit (SSU) ribosomal RNA in Dictyostelium discoideum," Current Genetics, vol. 36, no. 1-2, pp. 55-61, 1999.

[26] C. Barth, U. Greferath, M. Kotsifas et al., "Transcript mapping and processing of mitochondrial RNA in Dictyostelium discoideum," Current Genetics, vol. 39, no. 5-6, pp. 355-364, 2001.

[27] C. Barth, P. Le, and P. R. Fisher, "Mitochondrial biology and disease in Dictyostelium," International Review of Cytology, vol. 263, pp. 207-252, 2007.

[28] P. Le, P. R. Fisher, and C. Barth, "Transcription of the Dictyostelium discoideum mitochondrial genome occurs from a single initiation site," RNA, vol. 15, no. 12, pp. 2321-2330, 2009.

[29] D. J. Watts and J. M. Ashworth, "Growth of myxameobae of the cellular slime mould Dictyostelium discoideum in axenic culture," Biochemical Journal, vol. 119, no. 2, pp. 171-174, 1970.

[30] M. Darmon, P. Brachet, and L. H. P. Da Silva, "Chemotactic signals induce cell differentiation in Dictyostelium discoideum," Proceedings of the National Academy of Sciences of the United States of America, vol. 72, no. 8, pp. 3163-3166, 1975.

[31] M. Sussman, "Biochemical and genetic methods in the study of cellular slime mold development," Methods in Cell Biology, vol. 2, no. C, pp. 397-410, 1966.

[32] W. Nellen, C. Silan, and R. A. Firtel, "DNA-mediated transformation in Dictyostelium discoideum: regulated expression of an actin gene fusion," Molecular and Cellular Biology, vol. 4, no. 12, pp. 2890-2898, 1984.

[33] Z. Wilczynska and P. R. Fisher, "Analysis of a complex plasmid insertion in a photoaxis-deficient transformant of Dictyostelium discoideum selected on a Micrococcus luteus lawn," Plasmid, vol. 32, no. 2, pp. 182-194, 1994.

[34] P. R. Gilson, X.-C. Yu, D. Hereld et al., "Two Dictyostelium orthologs of the prokaryotic cell division protein FtsZ localize to mitochondria and are required for the maintenance of normal mitochondrial morphology," Eukaryotic Cell, vol. 2, no. 6, pp. 1315-1326, 2003.

[35] A. U. Ahmed, P. L. Beech, S. T. Lay, P. R. Gilson, and P. R. Fisher, "Import-associated translational inhibition: novel in vivo evidence for cotranslational protein import into Dictyostelium discoideum mitochondria," Eukaryotic Cell, vol. 5, no. 8, pp. 1314-1327, 2006.

[36] P. B. Bokko, L. Francione, E. Bandala-Sanchez et al., "Diverse cytopathologies in mitochondrial disease are caused by AMPactivated protein kinase signaling," Molecular Biology of the Cell, vol. 18, no. 5, pp. 1874-1886, 2007.

[37] M. R. Karpenahalli, A. N. Lupas, and J. Söding, “TPRpred: a tool for prediction of TPR-, PPR- and SEL1-like repeats from protein sequences," BMC Bioinformatics, vol. 8, article 2, 2007.

[38] T. S. Kroeger, K. P. Watkins, G. Friso, K. J. Van Wijk, and A. Barkan, "A plant-specific RNA-binding domain revealed through analysis of chloroplast group II intron splicing," Proceedings of the National Academy of Sciences of the United States of America, vol. 106, no. 11, pp. 4537-4542, 2009.

[39] J. Jacobs and U. Kück, "Function of chloroplast RNA-binding proteins," Cellular and Molecular Life Sciences, vol. 68, no. 5, pp. 735-748, 2011.

[40] C. C. Des Francs-Small, T. Kroeger, M. Zmudjak et al., "A PORR domain protein required for $\mathrm{rpl} 2$ and $\mathrm{ccmF}_{C}$ intron splicing and for the biogenesis of $c$-type cytochromes in Arabidopsis mitochondria," Plant Journal, vol. 69, no. 6, pp. 996-1005, 2012.

[41] J. M. Zapata, V. Martínez-García, and S. Lefebvre, "Phylogeny of the TRAF/MATH domain," Advances in Experimental Medicine and Biology, vol. 597, pp. 1-24, 2007.

[42] M. G. Claros and P. Vincens, "Computational method to predict mitochondrially imported proteins and their targeting sequences," European Journal of Biochemistry, vol. 241, no. 3, pp. 779-786, 1996.

[43] W. Witke, W. Nellen, and A. Noegel, "Homologous recombination in the Dictyostelium $\alpha$-actinin gene leads to an altered mRNA and lack of the protein," The EMBO Journal, vol. 6, no. 13, pp. 4143-4148, 1987.

[44] C. Barth, D. J. Fraser, and P. R. Fisher, "Co-insertional replication is responsible for tandem multimer formation during plasmid integration into the Dictyostelium genome," Plasmid, vol. 39, no. 2, pp. 141-153, 1998.

[45] K. Meierhoff, S. Felder, T. Nakamura, N. Bechtold, and G. Schuster, "HCF152, an Arabidopsis RNA binding pentatricopeptide repeat protein involved in the processing of chloroplast psbB-psbT-psbH-petB-petD RNAs," Plant Cell, vol. 15, no. 6, pp. 1480-1495, 2003.

[46] D. Sosso, S. Mbelo, V. Vernoud et al., "PPR2263, a DYW-subgroup Pentatricopeptide repeat protein, is required for mitochondrial nad5 and cob transcript editing, mitochondrion biogenesis, and maize growth," Plant Cell, vol. 24, no. 2, pp. 676691, 2012. 

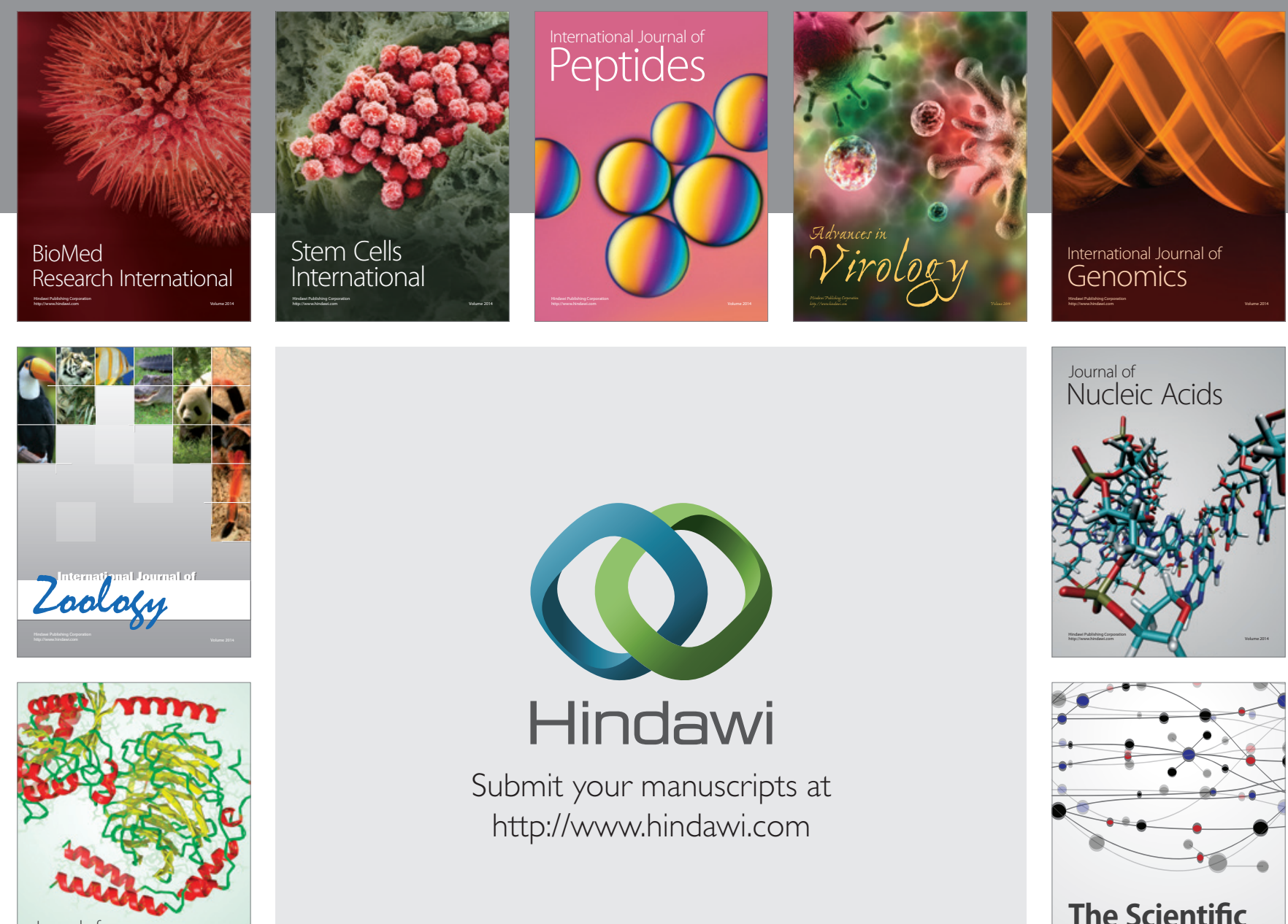

Submit your manuscripts at

http://www.hindawi.com

Journal of
Signal Transduction
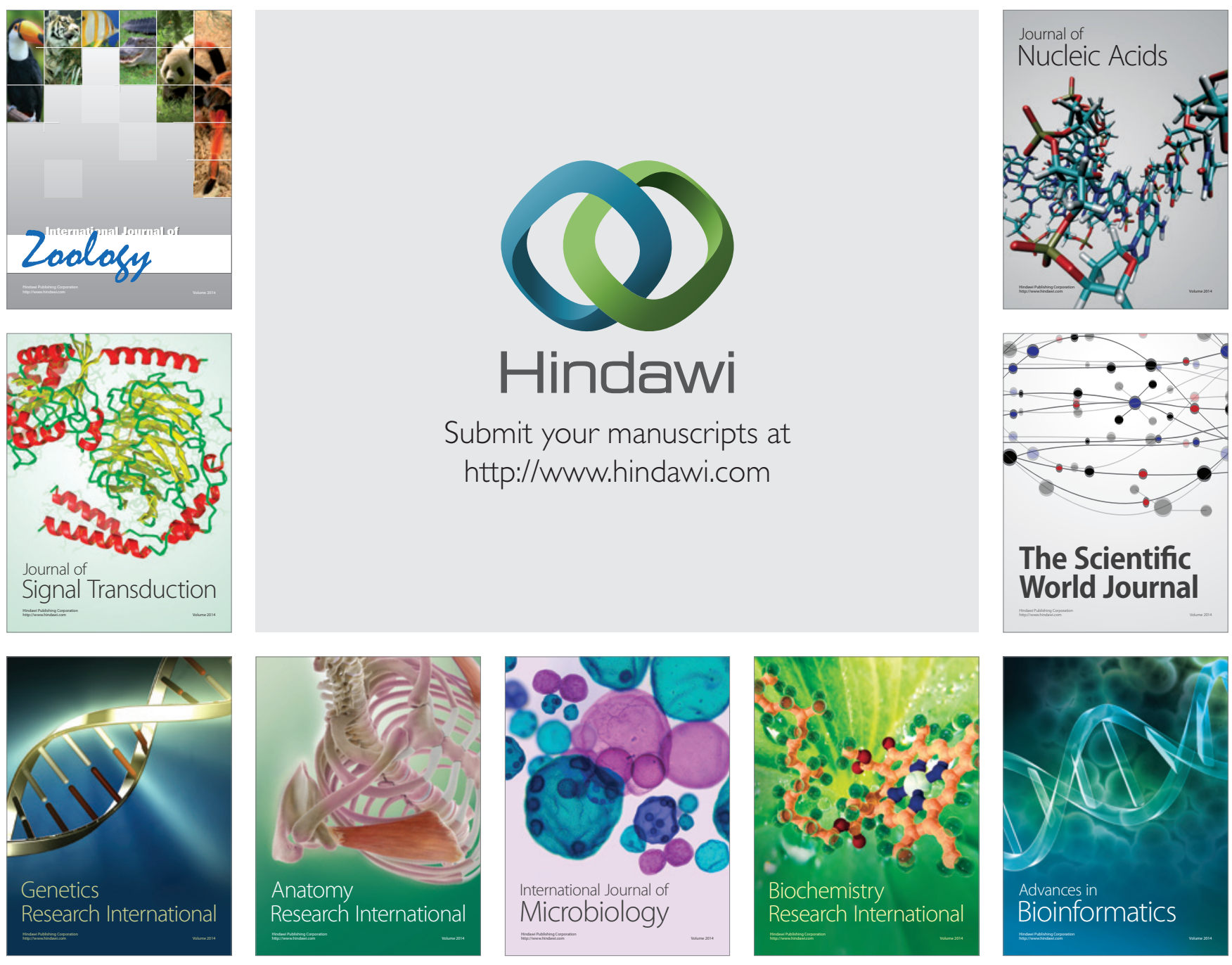

The Scientific World Journal
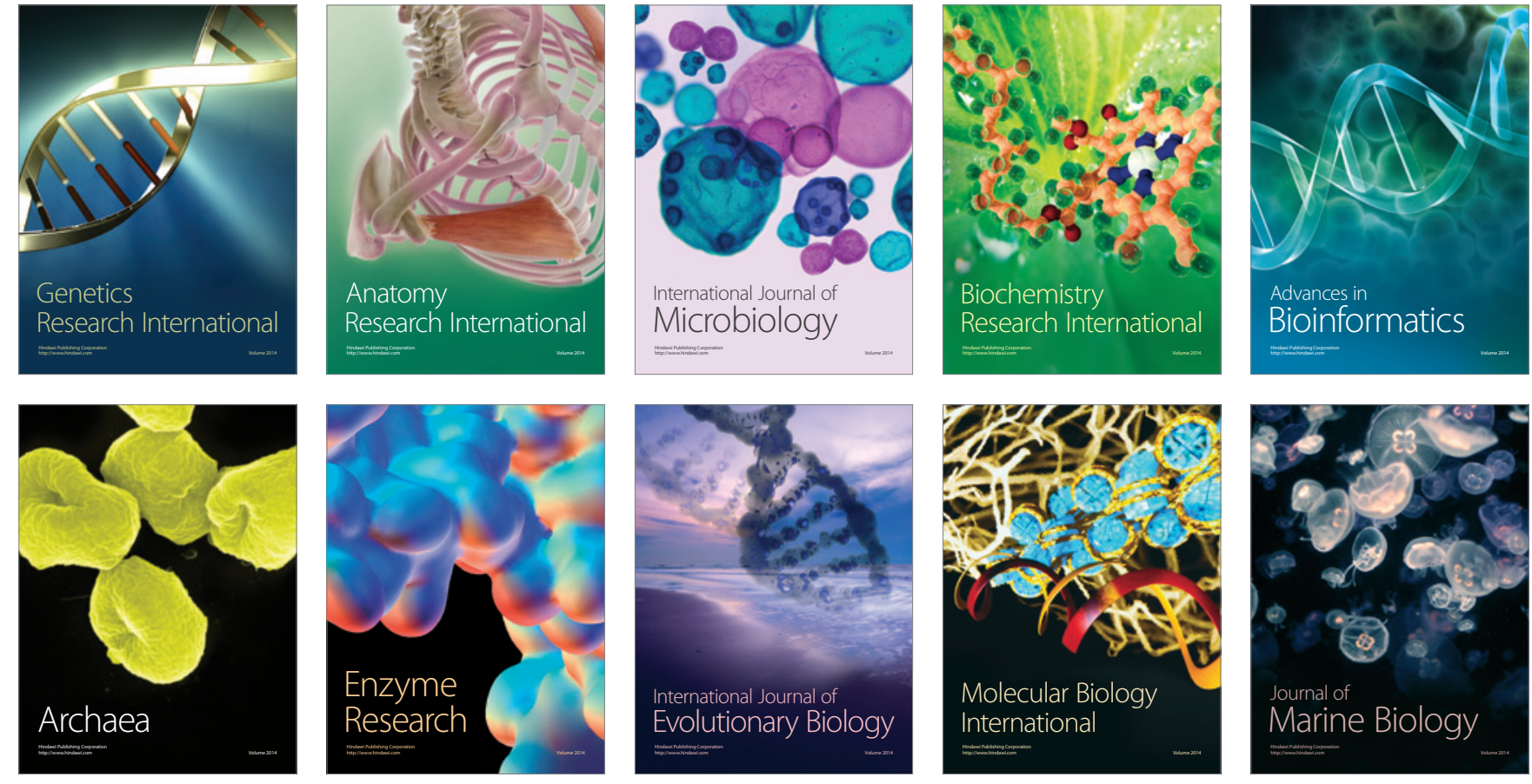